\title{
Rayleigh-Ritz Calculation of Effective Potential Far From Equilibrium
}

\author{
Francis J. Alexander ${ }^{a}$ and Gregory L. Eyink ${ }^{b}$ \\ a Center for Computational Science \\ 3 Cummington Street \\ Boston University \\ Boston, Massachusetts 02215 \\ $b$ Department of Mathematics \\ University of Arizona \\ Tucson, Arizona, 85721
}

(November 20, 2018)

We demonstrate the utility of a Rayleigh-Ritz scheme recently proposed to compute the nonequilibrium effective potential nonperturbatively in a strong noise regime far from equilibrium. A simple Kramers model of an ionic conductor is used to illustrate the efficiency of the method.

PACS. Numbers: 02.50.-r, 05.40.+j

We have recently proposed to use in nonequilibrium statistical mechanics a novel variational principle associated to the so-called effective action functional [1]. This quantity is well-known in quantum field theory, where the concept has it roots in the early work of Heisenberg \& Euler [3] and Schwinger [4] in QED. In nonequilibrium statistical mechanics, the first such action principle seems to have been Onsager's 1931 "principle of least dissipation" [5], which applies to systems subject to thermal or molecular noise, governed by a fluctuation-dissipation relation. A formulation of the least-dissipation principle by an action functional on histories was later developed by Onsager and Machlup [6]. In a weak-noise limit the field-theoretic effective action and the Onsager-Machlup action coincide, as discussed some time ago by Graham [7]. (See also [8]). However, the weak-noise limit is of rather restricted applicability. In particular, it is useless to deal with problems in which there is no small parameter and in which strong fluctuations dominate the phenomena on a wide range of length-scales. These include, for example, high Reynolds-number fluid and plasma turbulence, spinodal decomposition, and surface growth by random deposition. In strong-noise systems, efficient calculational tools remain to be discovered. One powerful nonperturbative scheme which was developed in quantum field theory is a Rayleigh-Ritz method based upon a constrained variation of the quantum energy-expectation functional 9 11. This method has been recently extended by us to nonequilibrium statistical dynamics with non-Hermitian evolution operator [1,2].

It is our purpose here to demonstrate the computational utility of that method in a simple concrete model, the Kramers model of an ionic conductor [12]. The model consists of a unit mass, charged particle in a 1dimensional cosine potential $V(x)=V_{0} \cos x$, damped by linear friction with coefficient $\gamma$, and driven both by an electric force $F=q E$ and by thermal noise of temperature $T=\gamma \Theta / k_{B}$. The dynamics of individual realizations is given by the nonlinear Langevin equation

$$
\ddot{x}+\gamma \dot{x}+V_{0} \sin x=F+\Gamma(t),
$$

with zero-mean Gaussian noise:

$$
\left\langle\Gamma(t) \Gamma\left(t^{\prime}\right)\right\rangle=2 \gamma \Theta \delta\left(t-t^{\prime}\right) .
$$

Here $x$ runs over $[0,2 \pi]$ with periodic b.c. Equivalently, the evolution of the single-time distribution $P_{t}$ of position $x$ and velocity $v=\dot{x}$ is given by the Fokker-Planck equation

$$
\begin{aligned}
\frac{\partial}{\partial t} P_{t}=-\frac{\partial}{\partial x}\left(v P_{t}\right)+ & \frac{\partial}{\partial v}\left[\left(\gamma v+V_{0} \cos x\right.\right. \\
& \left.\left.-F+\gamma \Theta \frac{\partial}{\partial v}\right) P_{t}\right] \equiv \hat{L} P_{t} .
\end{aligned}
$$

The important point for our discussion here is that all parameter values will be chosen order one, i.e. $V_{0}=$ $\gamma=F=\Theta=1$. In this case, there is neither a small nor a large parameter on which to base a perturbation expansion or asymptotic evaluation. In particular, $F=1$ implies that the system is far from thermal equilibrium, well beyond the regime of linear response of steady-state current $j(F)=\langle v\rangle_{F}$ to the applied field $F$.

In the statistical steady state, rather than the full effective-action, it is more useful to consider its timeextensive limit, the so-called effective potential. For any random variable $\mathbf{z}(t)$ in an ergodic system, this quantity measures the "cost" for fluctuations to occur in the empirical time-averages:

$$
\overline{\mathbf{z}}_{T} \equiv \frac{1}{T} \int_{0}^{T} d t \mathbf{z}(t)
$$

That is, it measures the probability for deviations to occur in a given realization at large $T$ between $\overline{\mathbf{z}}_{T}$ and the ensemble-mean $\overline{\mathbf{z}}$. Quantitatively, the relation between this probability and the effective potential function $V$ is 
that, for any possible single-time value $\mathbf{z}$ of the variable $\mathbf{z}(t)$, the probability for the average over a time-interval of duration $T$ to take on the value $\mathbf{z}$ has magnitude

$$
\operatorname{Prob}\left(\left\{\overline{\mathbf{z}}_{T} \approx \mathbf{z}\right\}\right) \sim \exp (-T \cdot V[\mathbf{z}])
$$

in the limit of large $T$. The potential function $V[\mathbf{z}]$ is nonnegative and convex, with minimum value $=0$ occuring only at the ensemble-mean, $V[\overline{\mathbf{z}}]=0$. The fluctuation formula Eq.(5) is a refinement of the standard ergodic hypothesis. It states not only that time-averages $\overline{\mathbf{z}}_{T}$ will converge to the ensemble-average $\overline{\mathbf{z}}$ almost surely in the limit of large $T$, but also it gives a precise quantitative estimate on the probability of the deviations. This refinement holds whenever some condition of finite exponential moments is satisfied: see [1,2]. The effective potential is analogous to the thermodynamic entropy of an equilibrium system (more precisely, to its negative) and Eq.(5) for the probability of statistical deviations is analogous to the Einstein-Boltzmann formula for the fluctuations away from equilibrium. This probabilistic interpretation of the effective potential was pointed out in field-theory by Jona-Lasinio [13]. Although any variable might be considered, we shall be interested here in the electric current $j$ and its effective potential $V[j]$.

The method we use to calculate $V$ is the RayleighRitz variational scheme proposed in [1.2]. As discussed in more detail there, the effective potential may be characterized very generally by means of a constrained variation of the "energy functional"

$$
\mathcal{H}\left[\Psi^{R}, \Psi^{L}\right] \equiv-\left\langle\Psi^{L}, \hat{L} \Psi^{R}\right\rangle,
$$

defined in terms of the Liouville operator $\hat{L}$ of the nonequilibrium statistical dynamics. $V[\mathbf{z}]$ was shown to be the extremal value of $\mathcal{H}\left[\Psi^{R}, \Psi^{L}\right]$ under arbitrary variation of "trial states" $\Psi^{R, L}$ subject to the constraints of constant overlap

$$
\left\langle\Psi^{L}, \Psi^{R}\right\rangle=1
$$

and constant means

$$
\left\langle\Psi^{L}, \hat{\mathbf{Z}} \Psi^{R}\right\rangle=\mathbf{z} .
$$

The operator $\hat{\mathbf{Z}}$ consists of multiplication by variable $\mathbf{z}$. By means of Lagrange multipliers $\lambda, \mathbf{h}$ associated to the constraints, this variational characterization of the effective potential can be expressed in spectral terms as

$$
V[\mathbf{z}]=\mathbf{z} \cdot \mathbf{h}-\lambda[\mathbf{h}],
$$

where $\lambda[\mathbf{h}]$ is the principal eigenvalue of the "perturbed" Liouville operator $\hat{L}_{\mathbf{h}}=\hat{L}+\mathbf{h} \cdot \hat{\mathbf{Z}}$. Observe that this spectral characterization is exactly analogous to the characterization of the free-energy in equilibrium statistical mechanics as the principal eigenvalue of the "transfermatrix" in lattice systems [14]. In that case, the entropy is then obtained by a Legendre transform of the free-energy, entirely analogous to the relation of $\lambda[\mathbf{h}]$ and $V[\mathbf{z}]$ in Eq.(9) by a Legendre transform. As the starting point for an approximate Rayleigh-Ritz calculation of $V$, any probability distribution function (PDF) ansatz for the stationary distribution of the nonequilibrium dynamics may be employed. The "right trial state" $\Psi^{R}(\mathbf{x})$ just consists of a guess for the stationary PDF, $\rho(\mathbf{x} ; \mathbf{c})$, depending upon some arbitrary parameters $\mathbf{c}$. The "left trial state" $\Psi^{L}(\mathbf{x})$ is a (linear superposition of a) set of selected moment-functions $\psi_{n}(\mathbf{x})$. The variation within such a framework leads in general to a "nonlinear eigenvalue problem" to determine the approximate value $\lambda_{\#}[\mathbf{h}]$ of the leading eigenvalue within the ansatz. See [2].

The simplest systematic approximation procedure in the Kramers model is to use the Gaussian trial weight

$$
w(x, v)=\frac{1}{\sqrt{2 \pi \Theta}} \exp \left[-\frac{v^{2}}{2 \Theta}\right],
$$

along with the corresponding truncated orthogonal polynomial expansions

$$
\Psi^{R}(x, v)=w(x, v) \cdot \sum_{n=0}^{N} \sum_{p=-P}^{P} c_{n, p}^{R} \cdot \frac{H e_{n}(v)}{\sqrt{n !}} \cdot \frac{e^{i p x}}{\sqrt{2 \pi}}
$$

and

$$
\Psi^{L}(x, v)=\sum_{n=0}^{N} \sum_{p=-P}^{P} c_{n, p}^{L} \cdot \frac{H e_{n}(v)}{\sqrt{n !}} \cdot \frac{e^{i p x}}{\sqrt{2 \pi}}
$$

Here $\mathrm{He}_{n}(v)$ are a set of Hermite polynomials, with conventions as in 15. The same expansion was used by Risken \& Vollmer [16] to calculate the full stationary measure. Orthogonal polynomial expansions have the simplifying feature that they lead to a linear problem for the approximate eigenvalue $\lambda_{\#}[h]$ and eigenvectors $\mathbf{c}_{\#}^{R}, \mathbf{c}_{\#}^{L}$ (with \# shorthand for $N, P$ ), as

$$
\sum_{n^{\prime}, p^{\prime}}\left(\mathbf{L}_{\#, h}\right)_{n p, n^{\prime} p^{\prime}} c_{\#, n^{\prime} p^{\prime}}^{R}=\lambda_{\#}[h] c_{\#, n p}^{R},
$$

and

$$
\sum_{n^{\prime}, p^{\prime}}\left(\mathbf{L}_{\#, h}^{\dagger}\right)_{n p, n^{\prime} p^{\prime}} c_{\#, n^{\prime} p^{\prime}}^{L}=\lambda_{\#}^{*}[h] c_{\#, n p}^{L},
$$

with

$$
\begin{array}{r}
\left(\mathbf{L}_{\#, h}\right)_{n p, n^{\prime} p^{\prime}}=\sqrt{(n+1) \Theta}(-i p+h) \delta_{p p^{\prime}} \delta_{n, n^{\prime}-1} \\
-n \gamma \delta_{p p^{\prime}} \delta_{n n^{\prime}}
\end{array}
$$

for $n=0, \ldots, N, p=-P, \ldots, P$ and $\left(\mathbf{L}_{\#, h}^{\dagger}\right)_{n p, n^{\prime} p^{\prime}}=$ $\left(\mathbf{L}_{\#, h}\right)_{n^{\prime} p^{\prime}, n p}^{*}$, the Hermitian conjugate. $\mathbf{L}_{\#, h}$ is the finite 
matrix approximation to the full "perturbed" operator $\hat{L}_{h}=\hat{L}+h \hat{V}$. The approximate value of the effective potential is then obtained from

$$
V_{\#}[j]=j_{\#}[h] \cdot h-\lambda_{\#}[h],
$$

in which $\lambda_{\#}[h]$ is the eigenvalue branch passing through 0 at $h=0$ and $j_{\#}[h] \equiv\left\langle\Psi_{\#}^{L}(h), \hat{V} \Psi_{\#}^{R}(h)\right\rangle$ is the associated "perturbed" current. The latter may be determined from the Hellmann-Feynman theorem [17], as $j_{\#}[h]=\lambda_{\#}^{\prime}[h]$. It can also be calculated directly from the approximate right and left eigenvectors, as

$$
\begin{aligned}
j_{\#}[h]=\sum_{n, p}\left[\sqrt{n}\left(c_{\#, n-1, p}^{L}\right)^{*} c_{\#, n, p}^{R}\right. & \\
& \left.+\sqrt{n+1}\left(c_{\#, n+1, p}^{L}\right)^{*} c_{\#, n, p}^{R}\right] .
\end{aligned}
$$

This avoids the evaluation of derivatives and is more computationally efficient in such a small system, where the determination of the eigenvalues and eigenvectors is easy to accomplish. These calculations have been carried out by us numerically, and the results for the approximate effective potential $V_{\#}[j]$ are graphed in Figure 1 for various choices of $N$ and $P$. As can be seen from that figure, convergence is already obtained in the range $j=0.2-1.4$ to $1 \%$ accuracy for $N=4, P=5$.

The effective potential can also be obtained by direct numerical solution (DNS) of the Langevin dynamics. The most obvious procedure would be to gather long time series of $v(t)$ in the steady-state, to assemble histograms of probabilities of empirical time averages $\bar{v}_{T}=\frac{1}{T} \int_{0}^{T} d t v(t)$ over time-intervals of duration $T$, and then to calculate $V[j]$ via the inverse to Eq.(5). However, we have found that the approximate potential from this method converges very poorly. Instead, the most efficient method is to determine approximate values of $\lambda_{\#}[h]$ and $j_{\#}[h]$ from

$$
\lambda_{\#}[h]=\frac{1}{T} \ln \left\langle\exp \left[h \int_{0}^{T} d t v(t)\right]\right\rangle
$$

and

$$
j_{\#}[h]=\frac{\left\langle\bar{v}_{T} \exp \left[h \int_{0}^{T} d t v(t)\right]\right\rangle}{\left\langle\exp \left[h \int_{0}^{T} d t v(t)\right]\right\rangle},
$$

where $\langle\cdot\rangle$ denotes average over some number $R$ of realizations and \# now stands for $R, T$ and the numerical time step $\Delta t$. Substituting these into Eq.(16), a value $V_{\#}[j]$ is obtained which for large $R, T$ and small $\Delta t$ converges to the true potential. We have also performed this direct calculation of $V[j]$ via a 2nd-order stochastic Runge-Kutta integration of the Langevin Eq.(1) for $R=32000, T=1000$ and $\Delta t=0.01$. Increasing $T$ or decreasing $\Delta t$ did not change the results, for which the largest errors are statistical, based upon the finite number of realizations $R$.

In Figure 2 we compare also the potentials $V[j]$ themselves for the above DNS and the $N=10, P=8$ Rayleigh-Ritz, over the range $j=0.718-0.733$. Over this range the error in the Rayleigh-Ritz value is at most one part in $10^{4}$. The relatively large error bars on $V_{\mathrm{DNS}}$ are due to the cancellation of linear terms in $h$ between $\lambda_{\#}[h]$ and $j_{\#}[h] \cdot h$ in Eq.(16). Because of this cancellation, getting an accuracy even to one part in $10^{2}$ for $V_{\#}$ required an accuracy to one part in $10^{4}$ for $\lambda_{\#}[h]$ and $j_{\#}[h]$. To achieve an accuracy of $V_{D N S}$ comparable to Rayleigh-Ritz would require a reduction in statistical error in DNS values of $\lambda_{\#}$ and $j_{\#}$ from 1 part in $10^{4}$ to 1 part in $10^{8}$. With this error estimated as $O\left(R^{-1 / 2}\right)$, it would require a computation time longer by a factor of $10^{8}$. The present calculation required $\sim 10^{12}$ floating-point operations (flops) and, on the machine we employed, took about $10 \mathrm{hrs}$, the bulk of which was devoted to generating random numbers. Thus, accuracy to one part in $10^{4}$ for $V_{\#}$ from DNS would require about $10^{5}$ years of computation! By contrast, the RayleighRitz computation with $N=10, P=8$ accurate to $0.01 \%$ required $\sim 10^{6}$ flops, performed in about 0.1 seconds. In general, the Rayleigh-Ritz evaluation of $V_{\#}[j]$ in the Kramers model requires for each value of $h$ a calculation of the leading eigenvalue $\lambda_{\#}[h]$ and its associated eigenvector for the tridiagonal matrix Eq.(15), which is an $O(N \cdot P)$ operation, and the calculation of current $j_{\#}[h]$ via the summation formula Eq.(17), which is also $O(N \cdot P)$. The number of flops required is $\sim 1000 N \cdot P$. The superiority of the Rayleigh-Ritz method compared with DNS is clear. Even more favorable is the fact that the Rayleigh-Ritz method gives a result as in Figure 1 over a range 100 times larger, to $1 \%$ accuracy, with only $N=4, P=5$. In this range errors in DNS due to finite $T$ and $R$ are very large because the $h$-weighted ensembles in Eqs.(18), (19) have a greater contribution from rare events as $h$ increases. Outside of a narrow range of current $j$ near the mean value the determination of effective potential by DNS is practically impossible.

In this work we have focused upon the Rayleigh-Ritz determination of the effective potential via a convergent scheme, the expansion in orthogonal polynomials. However, as discussed in detail in [2] any physically-inspired, nonlinear ansatz for the PDF or any "surrogate" random variables chosen to model the system variables may be used as well. This is important for systems with many degrees-of-freedom, such as high Reynolds number turbulence or large-scale dynamics of multiphase fluids, in which convergent schemes are presently not remotely feasible. In work in progress [18], we apply our variational methods to practical modeling of such systems, in particular hydrodynamic turbulence. Analogous time-dependent Rayleigh-Ritz methods are available to calculate the full effective action functional on histories: 
see [2]. There is some general similarity of our methods to the Hartee-Fock variational approximation applied by Crisanti \& Marconi [19] to the calculation of effective action in phase segregation dynamics. Both are intrinsically nonperturbative and capable of systematic improvement. In addition, the Rayleigh-Ritz scheme illustrated here has a very great flexibility to incorporate intuitive guesses into an analytical calculation: any PDF ansatz for the system variables whatsoever may be used as the basis for a calculation by our variational method.

Acknowledgements We would like to thank Y. Oono for useful discussions on this subject. Numerical simulations were carried out at the Center for Computational Science at Boston University and the Department of Mathematics at the University of Arizona.

[1] G. L. Eyink, "Turbulence noise," J. Stat. Phys., to appear June (1996).

[2] G. L. Eyink, "Action principle in nonequilibrium statistical dynamics," submitted to Phys. Rev. E (1996), chaodyn/9505001.

[3] W. Heisenberg and H. Euler, Z. Physik 98714 (1936).

[4] J. Schwinger, Phys. Rev. 82664 (1951).

[5] L. Onsager, (I) Phys. Rev. 37405 (1931); (II) 382265 (1931).

[6] L. Onsager and S. Machlup, Phys. Rev. 911505 (1953).

[7] R. Graham, in: Stochastic Processes in Nonequilibrium Systems. Sitges School, June 1978, eds. L. Garrido, P. Seglar, and P. J. Shepherd. Lecture Notes in Physics, vol. 84. (Springer-Verlag, Berlin, 1978).

[8] H. Calisto, E. Cerda, and E. Tirapegui, J. Stat. Phys. 82 1015 (1996).

[9] K. Symanzik, Commun. Math. Phys. 1648 (1970).

[10] J. M. Cornwall, R. Jackiw, and E. Tomboulis, Phys. Rev. D 102428 (1974).

[11] R. Jackiw and A. Kerman, Phys. Lett. A 71158 (1979).

[12] H. A. Kramers, Physica 7284 (1940).

[13] G. Jona-Lasinio, in: Scaling and Self-Similarity in Physics, ed. J. Fröhlich. (Birkhäuser, Boston, 1983).

[14] H. A. Kramers and G. H. Wannier, Phys. Rev. 60252 (1941); E. N. Lassettre and J. P. Howe, J. Chem. Phys. 9 252 (1941); E. W. Montroll, J. Chem. Phys. 9706 (1941).

[15] M. Abramowitz and I. A. Stegun, Handbook of Mathematical Functions. (National Bureau of Standards, Washington, D.C., 1964).

[16] H. Risken and H. D. Vollmer, Z. Physik B 33297 (1979); H. D. Vollmer and H. Risken, Z. Physik B 34313 (1979).

[17] E. Merzbacher, Quantum Mechanics. (John Wiley \& Sons, New York, 1961), Ch. 17.

[18] F. J. Alexander and G. L. Eyink, "Realizability, effective action, and predictive turbulence closures," in preparation (1996).

[19] A. Crisanti and U. M. B. Marconi, Phys. Rev. E 514237 (1995).

\section{FIGURE CAPTIONS}

Figure 1.) Approximate effective potential $V[j]$ for $\mathrm{N}=4, \mathrm{P}=5(\square), \mathrm{N}=6, \mathrm{P}=6(+)$ and $\mathrm{N}=10, \mathrm{P}=8(\diamond)$.

Figure 2.) Potentials $V[j]$ for DNS (with errorbars) and the $N=10, P=8$ Rayleigh-Ritz solid line). 


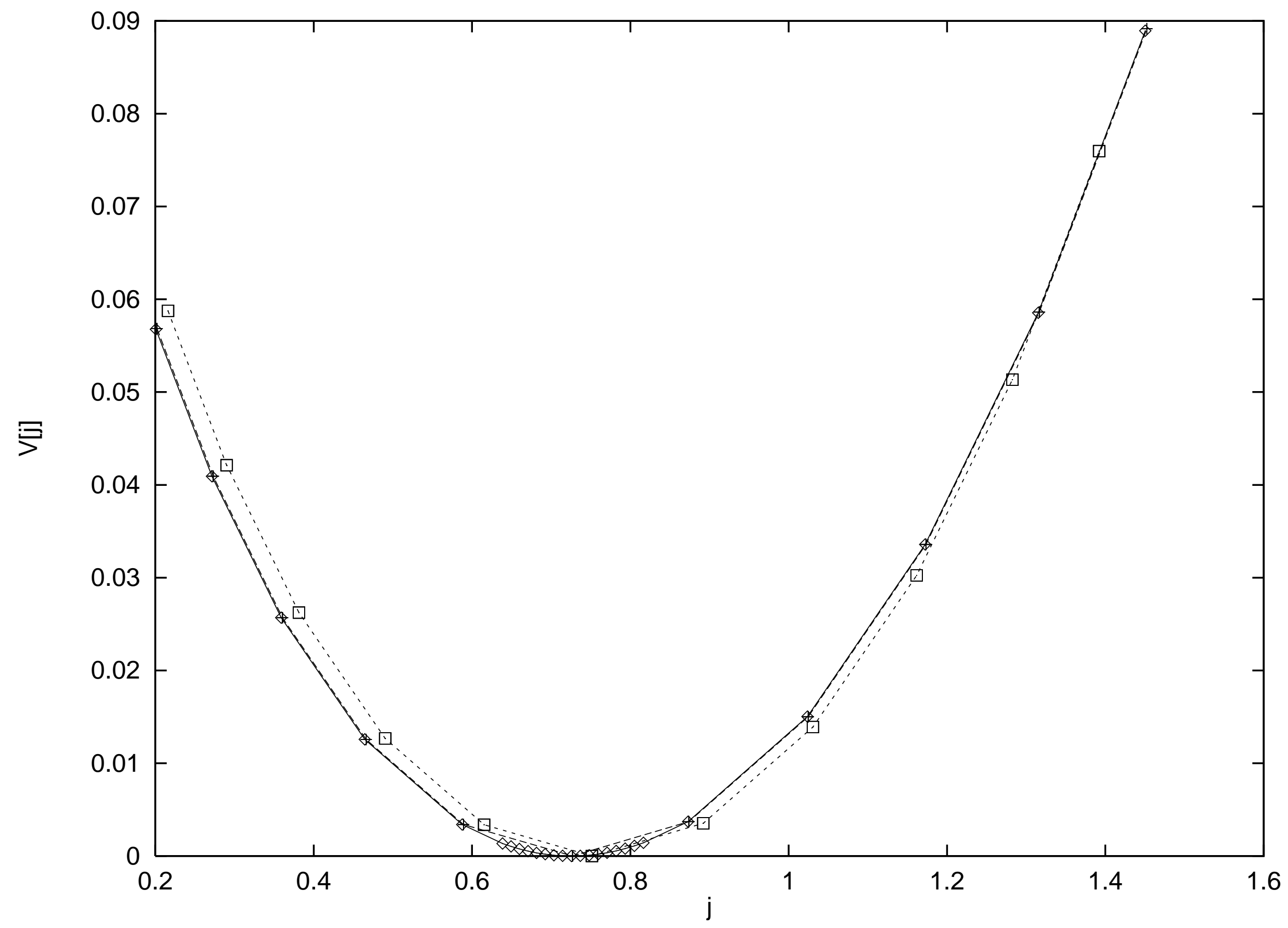




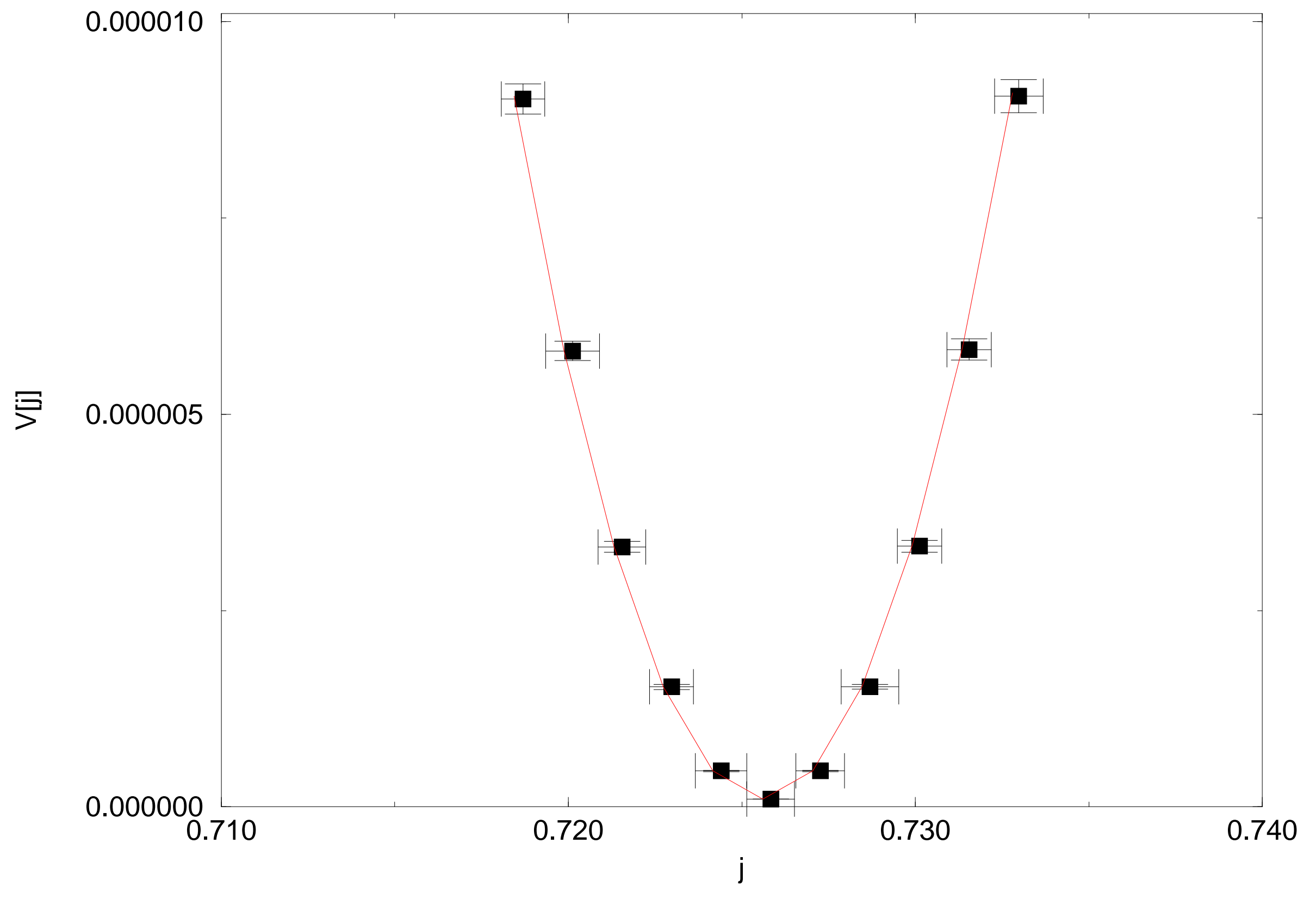

